\title{
CONF-970302--18
}

\section{A NEW PROCEDURE FOR MAKING TEM SPECIMENS OF SUPERCONDUCTOR DEVICES*}

\author{
Y. Huang and K. L. Merkle \\ Materials Science Division \\ Argonne National Laboratory \\ Argonne, IL 60439 USA
}

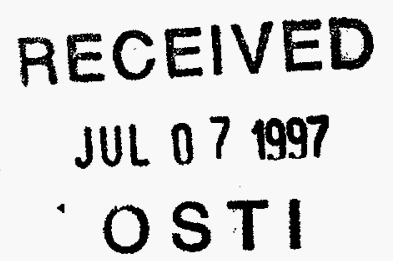

-

\section{April 1997}

The submitted manuscript has been created
by the University of Chicago as Operator of
Argonne National Laboratory ("Argonne")
under Contract No. W-31-109-ENG-38 with
the U.S. Department of Energy. The U.S.
Government retains for itself, and others
acting on its behalf, a paid-up, non
exclusive, irrevocable worldwide license in
said article to reproduce, prepare derivative
works, distribute copies to the public, and
perform publicly and display publicly, by or
on behalf of the Government.

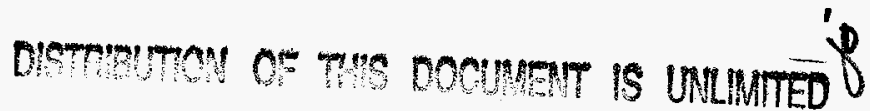

\section{DISCLAIMER}

\begin{abstract}
This report was prepared as an account of work sponsored by an agency of the United States Government. Neither the United States Government nor any agency thereof, nor any of their employees, makes any warranty, express or implied, or assumes any legal liability or responsibility for the accuracy, completeness, or usefulness of any information, apparatus, product, or process disclosed, or represents that its use would not infringe privately owned rights. Reference herein to any specific commercial product, process, or service by trade name, trademark, manufacturer, or otherwise does not necessarily constitute or imply its endorsement, recommendation, or favoring by the United States Government or any agency thereof. The views and opinions of authors expressed herein do not necessarily state or reflect those of the

United States Government or any agency thereof.
\end{abstract}

To be presented at the Materials Research Society, March 31-April 4, 1997, San Francisco, CA.

\footnotetext{
*Work supported by the U.S. Department of Energy, Basic Energy Sciences-Materials Sciences, under contract \#W-31-109-ENG-38 and the National Science Foundation, under contract \#DMR 91-20000.
} 


\section{A NEW PROCEDURE FOR MAKING TEM SPECIMENS \\ OF SUPERCONDUCTOR DEVICES}

Y. HUANG and K. L. MERKLE

Materials Science Division, Argonne National Laboratory. Argonne. II 60439

\section{ABSTRACT}

A new procedure is developed for making TEM specimens of thin film devices. In this procedure the sample is flatly polished to an overall "ion-mill-ready" thickness so that any point in the 2-D sample plane can be thinned to an electron-transparent thickness by subsequent ionmilling. Using this procedure small regions of interest can be easily reached in both cross-section and plan-view samples. This is specially useful in device studies. Applications of this procedure to the study of superconductor devices yield good results. This procedure, using commercially available equipment and relatively cheap materials, is simple and easy to realize.

\section{INTRODUCTION}

The goal of the sample preparation for transmission electron microscopy (TEM) is to obtain electron-transparent areas large enough to cover the microstructure to be observed. In the case of thin film device studies the small regions of interest (ROI) such as junctions need to be included in the thin areas. In the conventional dimpling method, the thin areas are obtained by grinding the sample with a wheel tool followed by ion-milling. Because the wheel tool can not be accurately positioned it is very difficult to align the dimpled area with the small ROI. Since the area which can be finally thinned to electron-transparent thickness is rather small, the possibility to include the ROI in the observable thin area is limited. In other preparation methods such as chemical etching the position of the thin area is even more uncontrollable.

Many efforts have been made to solve these problems. The precision tripod polishing method $[1,2]$ is the best solution so far. In this method the sample is polished into a wedged shape. The region near the wedge edge can be thinned to an electron-transparent thickness directly or by a short time of ion milling. The sample is mounted in a manner that the polishing process can be monitored by microscope and stopped when a ROI is in the thin regions. Since the entire edge is thinned, the observable area is relatively large and the ROIs to be observed can be readily reached. This method has been applied to prepare TEM samples of many materials and yields fairly good results[3-5]. However, the tripod method still has some limitations. First, since the sample is polished into a wedge, the eventually observable sample area is limited to the area near the edge. This may limit its application in samples in which the ROIs are not in a single line. Second, also because of the wedge shape it is not possible to polish multi-samples at the same time. This further limits any increase of the sample preparation efficiency. Third, the use of the expensive diamond lapping films in the tripod method makes the cost of preparation much higher than other methods. Also, this method is an experience-intense technique and not easy to be automated.

The procedure discussed in this paper was designed to make TEM samples of thin film devices. The difference of this new procedure compared to previous methods is that the entire 2D sample area can be made available for observation by flatly polishing it to an overall "ion-millready" thickness. The access to a specific ROIs is realized by subsequent ion-milling. The milling process can be monitored under either optical or electron microscope. This new

procedure is termed as "flat polishing method". Using this method the difficulty of observation of small junction regions in thin film devices can be easily overcome, relatively large thin areas can be obtained and most of the limitations of the tripod method can be eliminated.

\section{THE EXPERIMENTAL PROCEDURE}

\section{Obtaining cross-section pieces}

Fig. 1 schematically illustrates the procedures of the flat polishing method for TEM sample preparation. The first step of making cross-section samples is to embed the sample chip between two pieces of protective materials. Good protective materials should be strong and crack-resistant at small thickness. The materials need to be transparent if one wants to locate the ROI and 
measure the foil thickness with an optical microscope during preparation. For most of our work, slide glass is a good choice. Si is also a good material for the protective pieces because it is strong and can be used as a thickness indication at the final stage of polishing. The drawback is that $\mathrm{Si}$ is not transparent. The glue we used for embedding is M-600 epoxy which is cured at $120^{\circ} \mathrm{C}$ for $1-2$ hours. Cross-section pieces $0.2-0.5 \mathrm{~mm}$ thick are then cut from the cured sample block using a diamond blade saw or a wire saw. The advantage of the wire saw is that it wastes very little sample material and the finished surfaces are relatively good. Using a WS-2 wire saw made by Unipress, for example, we were be able to cut 5-6 cross-section pieces out of a 2-mm sample. The cutting direction is perpendicular to the junction lines or the boundary in the sample.

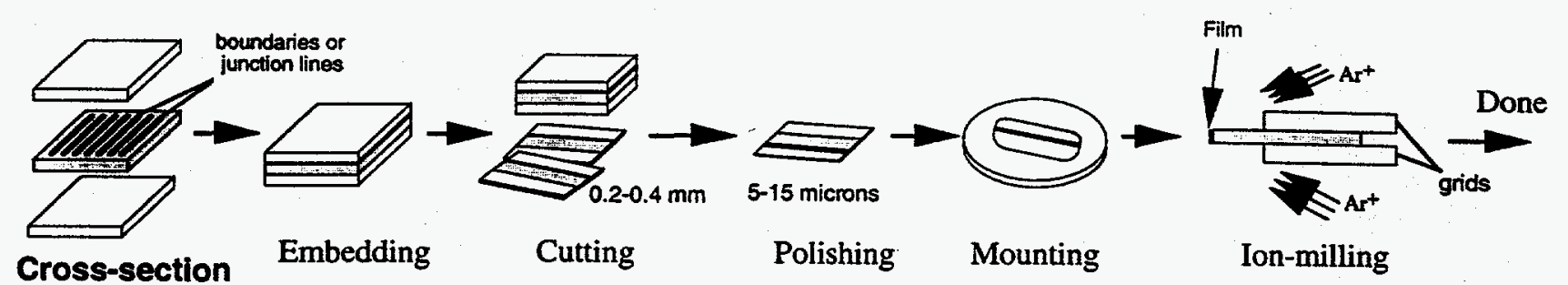
Samples
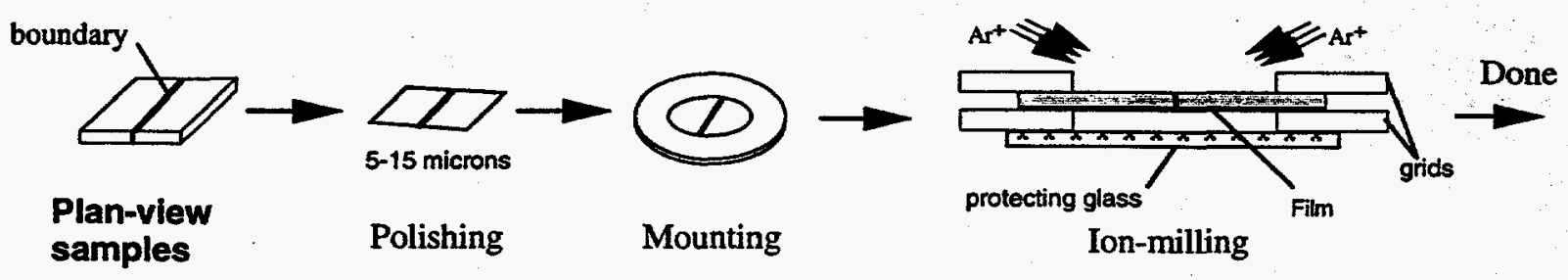

Figure 1. The procedures of the flat polishing method for TEM specimen preparation

\section{Polishing}

To get a good result in the polishing procedure the sample should be mounted carefully. We use CrystalBond wax for mounting. For a cross-section piece to be polished on both sides, the second mounting is the most critical to the quality of the final polish. For a thin film plan-view sample to be polished only on the substrate side, the chip can be directly mounted with the film side face down. Because the desired final thickness of the sample foil is about 10 microns, the thickness of the wax layer under the sample must be much smaller than 10 microns and should be uniform over the entire sample plane. To realize this small uniform thickness, the following measures need to be adopted. First, both the mount and the sample surfaces must be very clean, with no foreign particles mixed in the wax layer. Second, a uniform force need to be applied on the sample. Third, the mounting temperature should be adjusted to get a low viscosity of the mounting wax. This temperature should be kept until the force has been applied and the force should be kept until the sample is cooled. Fourth, if multi-samples are to be polished on a single mount, all the sample pieces need to have approximate the same starting thickness before mounting to ensure the uniform thickness of the wax layer.

Two kinds of polishing machines were used in our sample preparation. One is a Minimet with TEM preparation Kit, made by Buehler, Inc. This machine has a large disk-shaped sample holder ( $C$ in fig.2) sitting on the base A with a bearing $B$ between them. The sample $S$ is mounted on a small glass disk $M$ stuck by oil on the lower end of the screw $D$ at the center of the holder. When the holder is driven to move horizontally the sample is being polished by the polishing materials $\mathbf{P}$ on the base. The final sample thickness is controlled by the height of the screw D. Since the holder and the bearing is precisely engineered and relatively large in size the sample is kept straight and polished flatly and uniformly.

Another machine is a LabOne polishing and lapping system, made by $\mathrm{Mr}$ Semicon, Inc. This machine has a zero-vibration belt-driven 8 " polishing disk (fig.3) and a flatness control jig. 


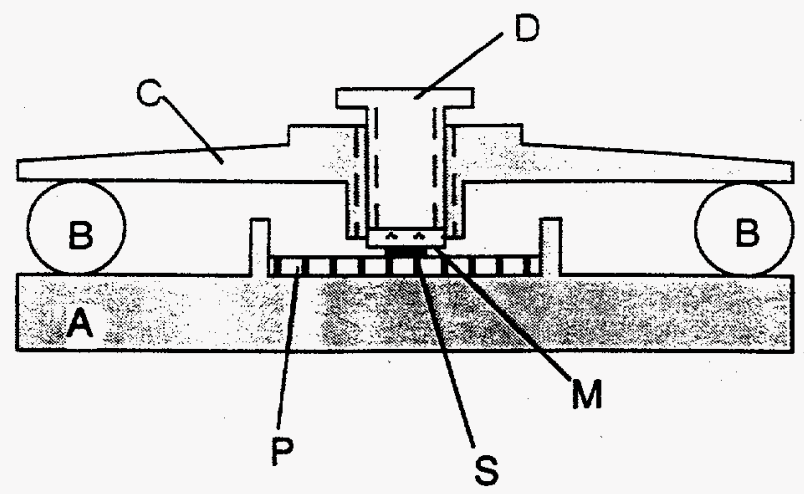

Figure 2. TEM preparation setting of Minimet polishing machine made be Buehler, Inc.

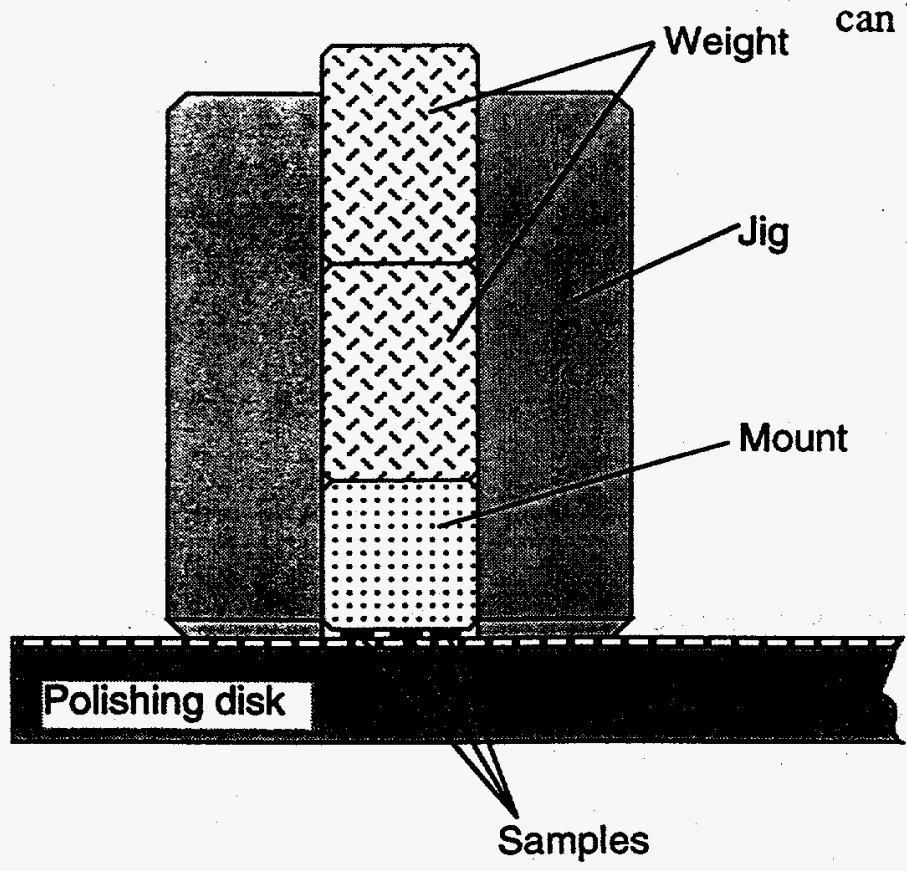

Figure 3. Flatness control jig of the LabOne polishing system made by MrSemicon, Inc.

thickness down and 3 and 1 micron pastes are used to get a smooth surface. Because soft polishing materials are used the sample can be polished to a small thickness without cracking relatively easily. In principle, the smaller the final thickness, the better the TEM samples. But when the foil becomes very thin, cracks still develop at the edge and the sample could be damaged. The appropriate final thickness depends on the brittleness of the substrate materials. For strong material such as $\mathrm{Si}$ or $\mathrm{SrTiO}_{3}$, it could be as small as 5 microns, but for brittle materials such as $\mathrm{LaAlO}_{3}$ the final thickness could be larger than 15 microns.

One way to prevent cracking in the final stage of polishing is to protect the sample edge by glue. We use Super Glue which is a kind of 1-minute fast glue widely used for everyday purpose. This glue is suitable for the edge protection purpose because it is significantly stronger than CrystalBound and is able to be dissolved in acetone. Before applying the glue the wax on the sample and the mount surfaces need to be removed. This can be done by a soft brush with acetone. A layer of the Super Glue is then applied on the mount with all the sample edges covered. By protecting the sample edge this way, the cracking is significantly reduced and the flatness of the sample is improved. In principle, one can directly use the Super Glue to mount the sample. However, because the glue solidifies so fast an unwanted thick glue layer is formed between the sample and the mount before the force is applied. The problem is more serious when 
mounting multi-samples. To solve this problem, we need a kind of glue which is similar to the Super Glue but with lower viscosity and longer solidifying time. If we can find a glue like this the final thickness of the polished sample could be further decreased and the quality of the TEM sample could be further improved.

\section{Ion milling}

The polished sample foil is lifted from the mount by dissolving the wax and glue in acetone. The foil is so fragile that it can only be carried on a piece of paper and moved with the tip of hair. To be able to handle it easily the foil needs to be mounted on a grid. The grids we used are $3 \mathrm{~mm}$ Mo grids with a single hole or a $2 \times 0.5 \mathrm{~mm}$ slot. For cross-section sample the slot grids are preferred because they allow as long as $2 \mathrm{~nm}$ of cross-section area to be thinned uniformly. For plan-view samples single-hole grids are used. We did not use $\mathrm{Cu}$ and $\mathrm{Ni}$ grids because the former could interfere with the analysis of $\mathrm{Cu}$ and the latter could lead to extra astigmatism.

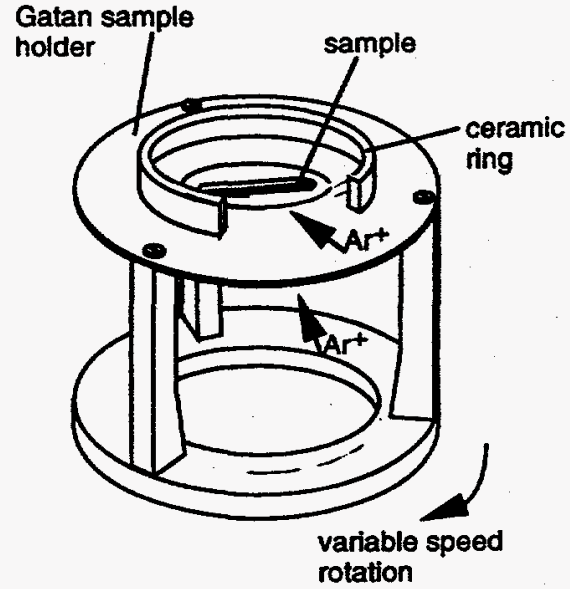

Figure 4. Modified Gatan ion-mill sample holder
The sample foil is then ion-milled to get electrontransparent thickness. The ion-mill we used is a Gatan DuoMill 600 . The modified sample holder for crosssection samples is shown in fig.4. By using the sector speed control along with the ceramic pieces attached to the sample holder for shielding, the cross-section samples are milled from the back side of the films only (see fig.1). The shadow effect of this milling geometry ensures an electron-transparent thickness to be obtained over a large area. It also minimizes the preferential thinning and gives relatively uniform thin areas. A typical milling condition is $4 \mathrm{keV}$ of ion beam energy and $18^{\circ}$ of tilt angle. In the final stage lower ion beam energy and smaller tilt angles are used.

Under a normal condition, the ROIs on the crosssection line are thinned first and hence the thinnest areas

are at the sample edge. Fig.5a is an optical micrograph which shows such a normally milled sample. This image was taken under monochromatic light so that the thickness contour fringes formed by the light interference can be seen clearly. The fringes nearly parallel to the edge indicate the monotonic thickness decrease from the grid edge to the sample edge, which is desirable for a good sample. Sometimes, however, the thickness decrease from the grid edge to the sample edge is not monotonic: the middle area is thinned faster than the edge. This leads to a situation as in fig. $5 \mathrm{~b}$, where the region on line $\mathrm{B}$ is thinner than the cross-section line $\mathrm{A}$. The perforation in area B will finally result in loss of the ROIs. To avoid this undesired result, the ROI should be mounted closer to the grid edge. Typically, for a $40 \mathrm{~mm}$ Mo grid and $18^{\circ}$ tilting angle the sample foil should be mounted so that the cross-section line is at about 130 microns from the grid edge. This can be done with the help of an optical microscope. If for some reason the situation shown in fig.5b has already occurred, to avoid loss of ROI in the further ion-milling some glue can be applied to protect the middle area. Care must be taken to make sure that the ROIs are not covered by the glue.

For the transparent substrates such as $\mathrm{MgO}$ and $\mathrm{LaAlO}_{3}$, the approach to an electrontransparent thickness can be detected by observing color fringes in the thin areas by optical microscopy under white light. Usually, one or two places on the cross-section line reach the electron-transparent thickness first. This is adequate for observation if these thinned areas represent the structure of the rest of the cross-section line. If the initial thinned areas are not the ROI to be observed, one can always mill the sample more to bring the ROI to a electrontransparent thickness. After the first cycle of observation the sample can be milled again to bring more ROIs on the cross-section line to electron-transparent thickness for more observation. Working this way the observation of isolated small devices become much easier than with the dimple method. Even in the case of an actual SQUID sample where only a single ROI is present in the whole cross-section line, it is still possible to find it by carefully milling the sample while monitoring by microscope. This process may be time-consuming because the milling-observing 


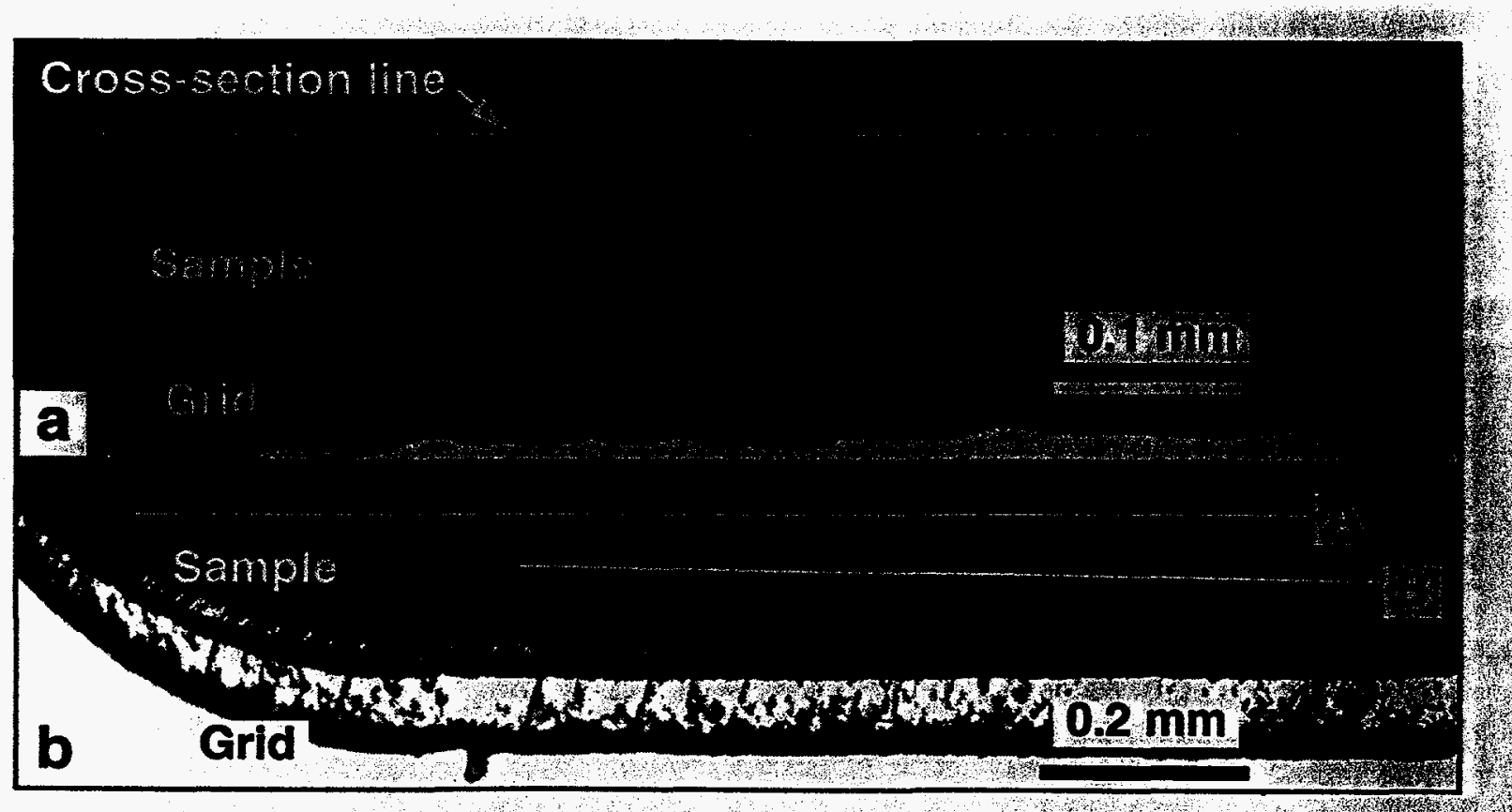

Figure 5 Optical micrograph of cross-section samples taken under monochromatic light. In sample a) the thinnest region is at the edge; in sample b) the regions on line $B$ are thinner than regions on the crosssection line $A$ at the edge, which is undesirable.

cycle may need to be repeated many times. Nevertheless, the study of the specific single region of a couple of microns in size becomes possible. To make this process easier a better optical microscope is needed on the ion-mill. Or if one can do the ion milling in situ in an electron microscope the observation of devices could be much faster and easier.

In the case of thin film plan-view samples, the ion milling is performed by a single ion gun from the substrate side. The film side could be protected from deposition of the sputtered materials by a piece of cover glass glued on the mill stage. After a hole formed, however, this glass piece should be removed because glass may be sputtered onto the film side by the ion beam through the hole. Normally, the ion milling is stopped immediately after perforation of the sample, but if no ROI is present in the initial thin regions, one can mill the sample for longer time to make the hole larger so that an ROI is reached at the edge of the hole. Similar to the case of cross-section samples, one can always mill the sample more to find other fresh ROIs for a new cycle of observation, except now the extension of the observable regions is in all the directions of the 2-D sample area.

\section{RESULT}

The procedure discussed above has been used to prepare TEM samples of $\mathrm{YBa}_{2} \mathrm{Cu}_{3} \mathrm{O}_{7-\mathrm{x}}$ (YBCO) superconductor devices, including superconductor-normal-metal-superconductor (SNS) edge junctions, superconductor-insulator-superconductor (SIS) junctions and several kinds of grain boundary junctions (GBJs). The materials of substrate and buffer layer include $\mathrm{MgO}$, $\mathrm{LaAlO}_{3}, \mathrm{SrTiO}_{3}, \mathrm{CaTiO}_{3}, \mathrm{CeO}_{2}$ and yttrium stabilized zirconia (YSZ).

The SNS junctions in the "edge" geometry (fig.6) is one of the promising implements for superconductor devices. The junctions studied in this work were fabricated by means of laser ablation and ion-beam etching techniques[6]. The main purpose of the TEM investigation is to reveal the interfacial microstructure of the junction regions. To facilitate the TEM observation an array of "junction lines" is specially made on a substrate. The direction of the lines is chosen to be a major low-index direction of the substrate, which makes HREM of the cross-section easier. In the cross-section sample the junction lines become isolated "junction points". When the spacing between these points is 40 microns or larger the use of the dimpling method becomes very difficult: it is very hard to find a junction in the limited thin area. There is no such limit in the flat polishing procedure. Because the entire cross-section line is polished, all junctions on the 


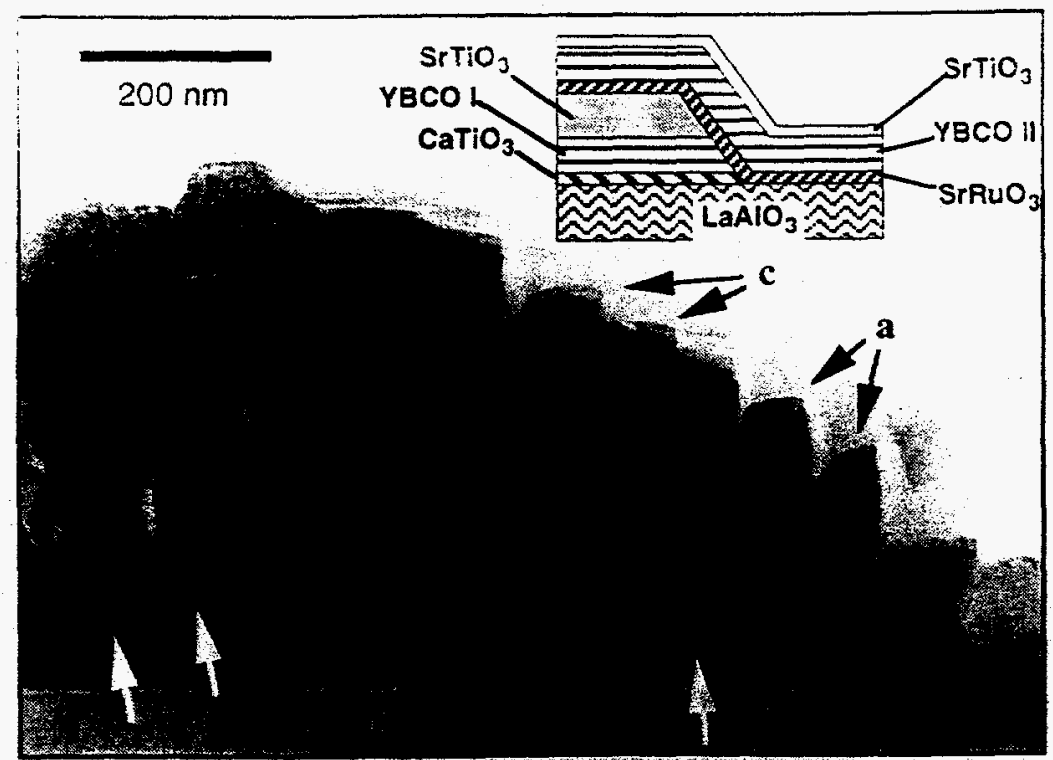

Figure 6. A TEM image of a sNs junction. The inset shows the designed geometry of the junction cross-section line can be thinned to electron-transparency. The number of observable junctions is much larger than in the dimpling method. The width of the thin area is also relatively larger. Fig. 6 shows a TEM image of an SNS junctions sample made by the flat polishing method. The quality of the sample is quite good. The various parts in the junction structure can be easily identified by their different contrast in the image. Extended defects such as a-axis particles and cracks in $\mathrm{SrTiO}_{3}$ layer and YBCO II layer can be clearly seen. Fig. 7 is a HREM image of a YBCO/SrRuO ${ }_{3}$ / YBCO junction area where the highly stepped SrRuO3 barrier layer is clearly observed.

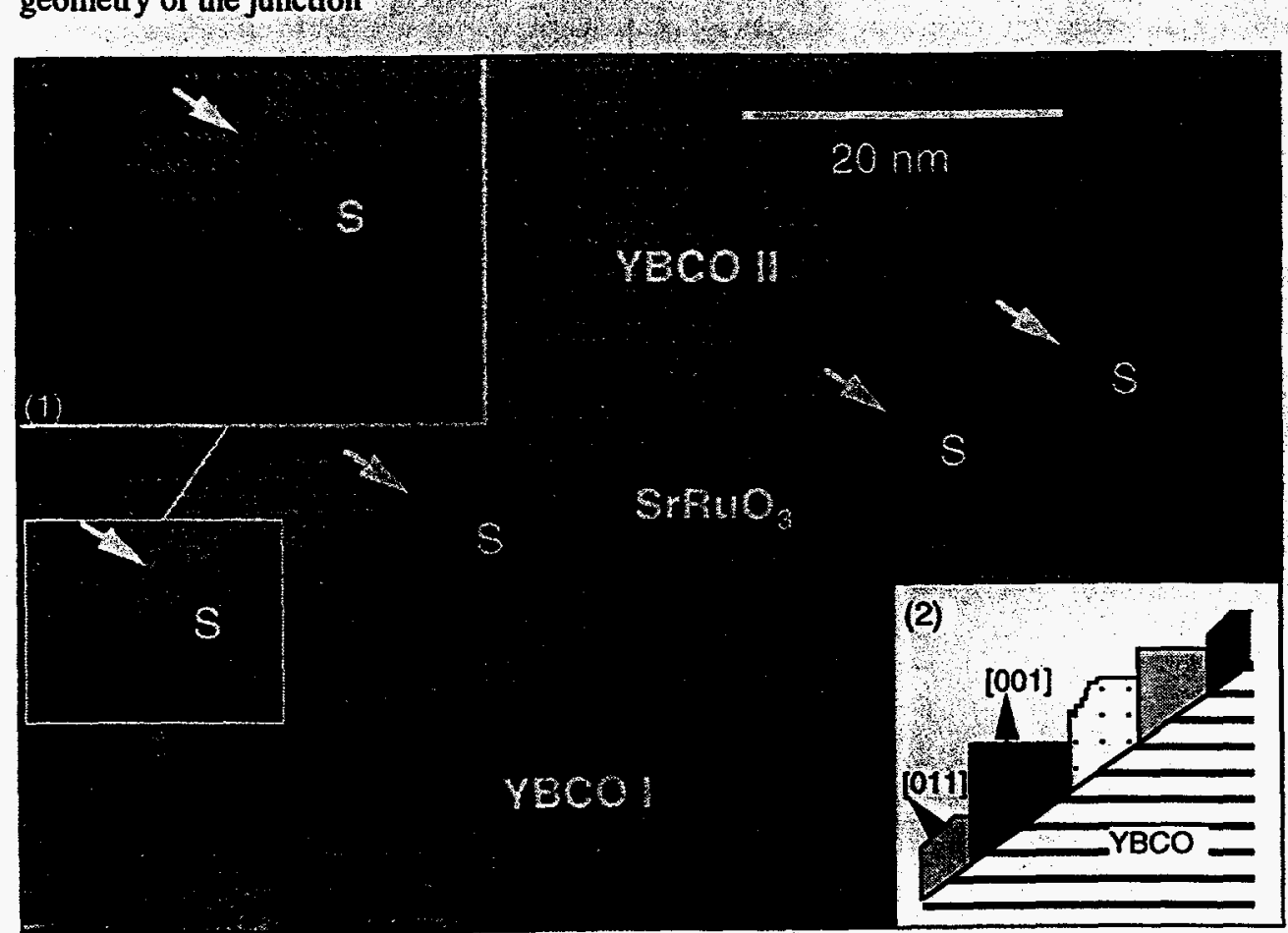

Figure 7. HREM image of a $\mathrm{YBCO} / \mathrm{SrRuO}_{3} /$ YBCO edge junction showing the highly stepped barrier layer. Inset 1 shows the detail of a step. Inset 2 illustrates the mechanism of the step formation.

One kind of GBJ studied in this work was made by the sputter-induced epitaxy modification (SIEM) method[7, 8], When fabricating this kind of junction a part of the MgO substrate is sputtered with a low-energy ion beam and then a layer of YBCO film is epitaxially grown. The in-plane orientations of the YBCO films grown on the sputtered and non-sputtered regions differ by $45^{\circ}$ and hence a $45^{\circ}$ tilt $\mathrm{GBJ}$ is formed between these two regions (fig. 8 ). The purpose of the microscopy is to study the property-microstructure relation of this artificial GBJ and to find out the mechanisms of the SIEM phenomenon by looking for differences in YBCO/MgO interfaces in the sputtered and non-sputtered regions. Similar to the case of SNS junctions, an array of the $45^{\circ}$ tilt grain boundary is made on an MgO substrate. Fig. 9 shows an HREM image of such a boundary. The structure of the boundary is quite complex: the starting point of the boundary is not at the edge of the sputtered region; the boundary is initially inclined but later straightens up and becomes a pure tilt boundary at some distance from the substrate. The starting point and 
inclination angles are different from boundary to boundary. In order to find the general features of these boundaries. larger numbers of boundaries need to be observed. By making the boundary array and using the flat polishing method this can be easily realized in a single TEM sample. In a study of the YBCO/MgO interfaces, remarkable differences in atomic-scale structure between sputtered and non-sputtered regions were observed(fig.10) An intermediate layer 3-6 nm thick is observed at the sputtered interface, which is identified as a $\mathrm{Bax} \mathrm{Mg}$ 1-x $\mathrm{O}$ layer formed by $\mathrm{Ba}$
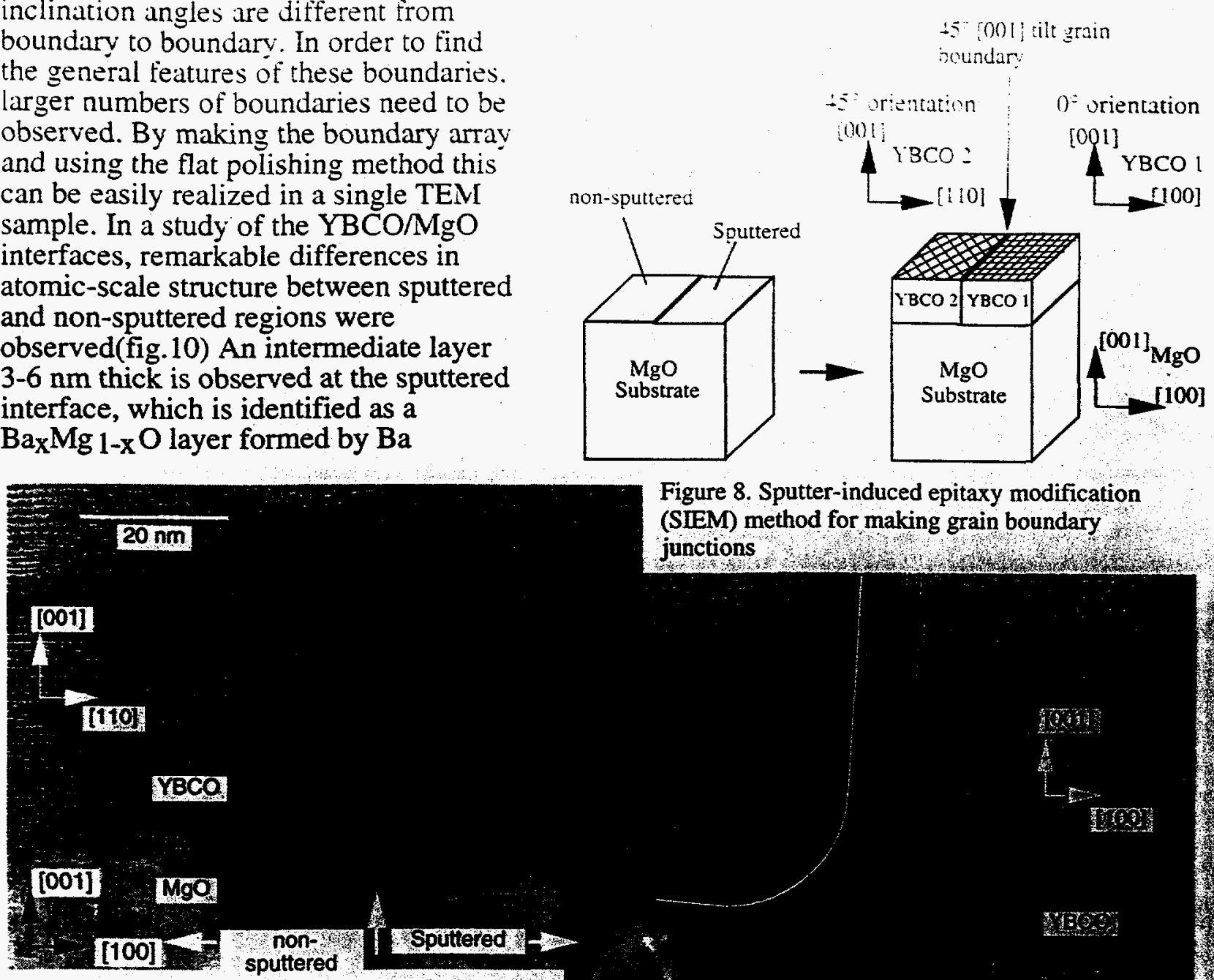

Figure 9. HREM image of an artificial grain boundary in YBCO film formed by SIEM method on $\mathrm{MgO}$ substrate. The boundary is outlined by a white curve. The YBCO/MgO interfaces are different in the sputtered and non-sputtered regions.

diffusion caused by the ion-sputtering. By using the flat polishing method long lengths of interfaces could be observed so that the conclusion about this intermediate layer is more reliable.

Another kind of GBJ is a bicrystal GBJ. The samples are actual SQUID devices with a single boundary. The purpose of the study is to find out the relation between the microstructure and the noise performance of the devices. Using the flat polishing procedure the preparation of the planview sample is rather simple. The chip is polished and milled on the substrate side until a boundary area is thinned at the edge of a hole. Fig. 11 shows plan-view images of boundary regions in high and low noise samples. A common feature of these two boundaries is that they meander across the straight line defined by the bi-crystal substrate. The structure of the films and boundaries are otherwise significantly different. In the high noise sample a lot of a-axis particles were observed. It is believed that the existence of the a-axis particles is the origin of the high noise because they not only cause large numbers of defects and a-c boundaries in the c-axis film but also result in a highly defective junction boundary. The cross-section TEM samples of these GBJs are relatively difficult to make. We have to rely on electron microscopy to locate the only region of interest on the entire cross-section line because it is not visible under optical microscope. The sample is first ion milled to a thickness at which electron diffraction pattern can be obtained. Since the orientation of the substrate are different in the two sides of the boundary, 


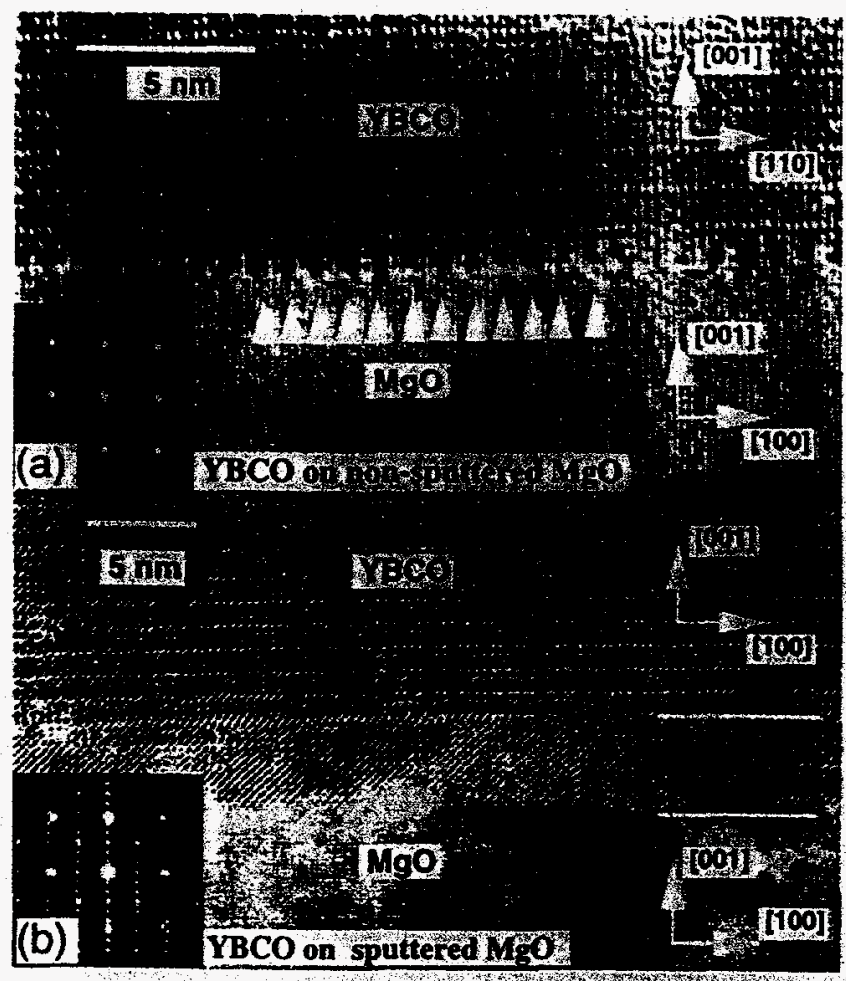

Figure 10. YBCO/MgO interfaces a) on non-sputtered substrate and b) on sputtered substrate.

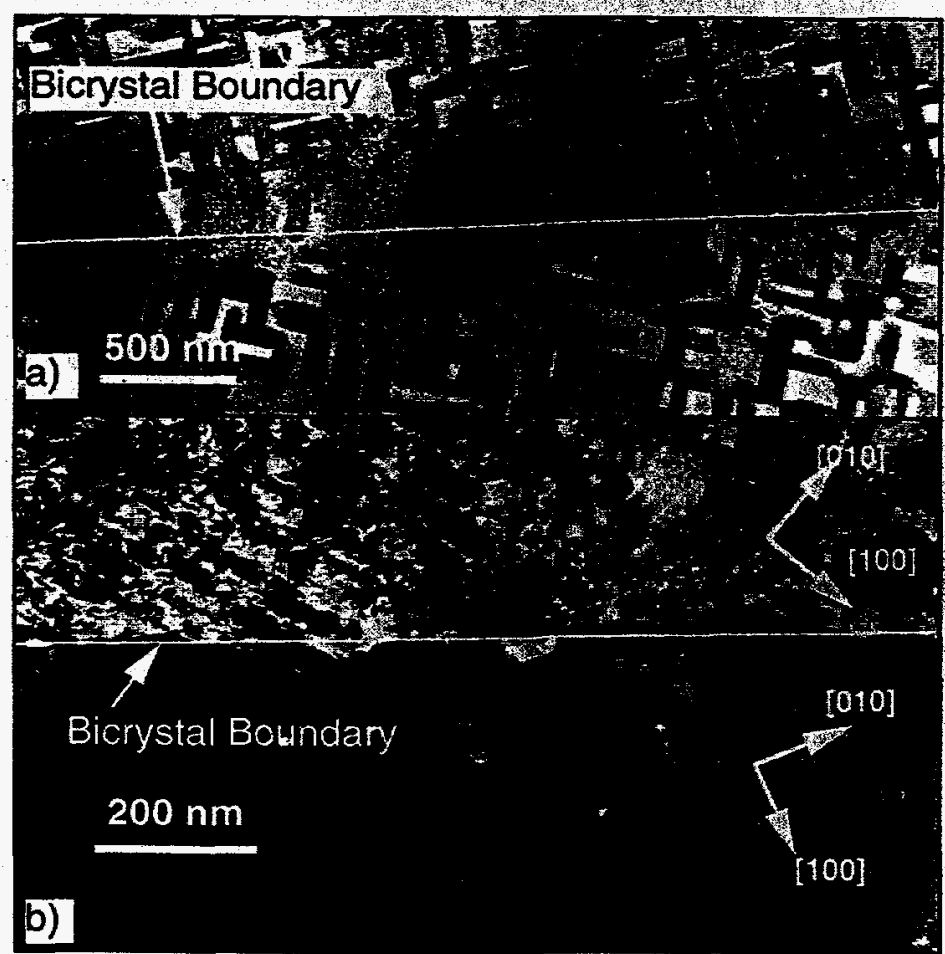

Figure 11. TEM images showing large difference in microstructure of the films and the boundaries between a) the high-noise device and $b$ ) the low-noise device. the position of the boundary can be found by electron diffraction. Fig. 12 is a cross-section image of the boundary in a high-noise sample. Some a-axis particles can be seen. The boundary in the YBCO film is a pure tilt boundary but it does not align with the bicrystal boundary. This is caused by the boundary meandering.

\section{CONCLUSION}

The examples discussed above indicate that the flat polishing method is a viable method for preparing TEM samples of thin film devices. This method has several advantages. First, by using this method maximum access to the ROIs in the sample can be realized. In the case of cross-section samples, similar to the result obtained by the tripod method, the entire cross-section line is made available for observation. In the case of plan-view samples, this method is better than the tripod method because any ROI in the 2-D sample plane can be reached for observation. The observable thin area is larger than with other methods. Thus, the study of isolated devices becomes possible. Second, this method is relatively simple and easy to learn. It can be used on most commercial precision polishing tools for TEM sample preparation, automated or manually-operated, as long as the flatness of the tool is better than a couple of microns. The use of soft cloth in the final polishing allows much larger tolerance to vibration of the polishing machine, to the brittleness of the sample materials and to the experience of the operator. Therefore, it can be used for a wider range of materials and the automation of the polishing process is relatively easy. Third, the sample preparation cost of using this method is much lower than the tripod method because it does not use the expensive diamond lapping sheet. Using existing equipment and saving in training expense also help lower the cost.

The disadvantage of the flat polishing method is that it needs longer ion-milling times for final thinning than that for the tripod method, although it is shorter than that for the dimple method. Therefore, the time saved in the

polishing process is largely offset by extra ion-milling time. Long-time ion-milling may cause some artifacts, such as preferential milling and formation of amorphous phases, etc. This causes special problems in the preparation of ion-beam sensitive materials. 


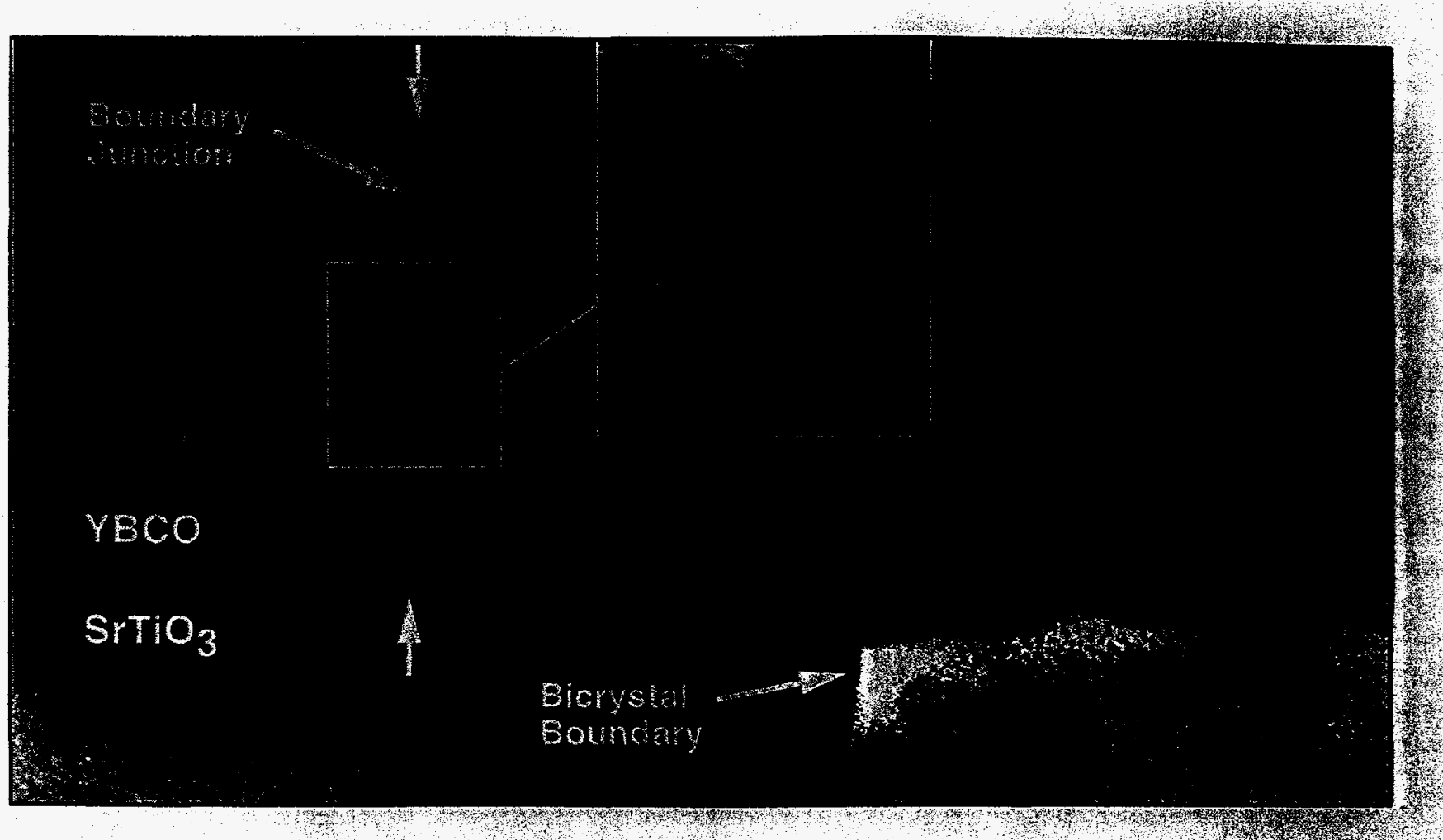

Figure 12. Cross-section TEM image of a grain boundary junction in a bicrystal SQUID. The boundary in the YBCO film does not align with the bicrystal boundary.

\section{ACKNOWLEDGMENT}

The authors are grateful to R.Csencsits, H.Zhang, and W.Sinkoer for their help in the HREM experiments. This work is supported by the National Science Foundation, under contract \#DMR 91-20000 (YH,) and the U. S. Department of Energy under contract \#W-31-109-ENG-38 (KLM)

\section{REFERENCES}

1. S. J. Klepeis , Mat. Res. Soc. symp. Proc., 115 (1988) 179.

2. J. Benedict, R. Anderson and S. J. Klepeis, Mat. Res. Soc. symp. Proc., 254 (1992) 121-140.

3. G. J. Wen, C. Traeholt, H. W. Zandbergen, K. Joosse, E. M. Reuvekamp and H. Rogalla, Phyaica C, 218 (1993) 29.

4. J. Ayache and P. H. Albarede, Ultramicro, 60 (1995) 195-206.

5. D. F. Dawson-Elli, M. A. Turowski, T. F. Kelly, Y.-W. Kim, N. A. Zreiba, .-M. Teng and Z. Mei, Mat. Res. Soc. symp. Proc., 199 (1990) 75-84.

6. Y. Huang, Microsc. Microansl., 3 (1997) in press.

7. B. V. Vuchic, K. L. Merkle, K. A. Dean, D. B. Buchholz, R. P. H. Chang and L. D. Marks, J. Appl. Phys., 77 (1995) 2591-2594.

8. Y. Huang, B. V. Vuchic, P. Balso, K. L. Merkle, D. B. Buchholz, S. Mahajan, J. S. Lei, P. R. Markworth and R. P. H. Chang, Mat. Res. Soc. Symp. Proc., 458 (1996) in press. 


\section{DISCLAMMER}

Portions of this document may be illegible in electronic image products. Images are produced from the best available original doccoment. 